\title{
Carbonate sludge as a nanostructured material for environmental engineering
}

\author{
Anastasiya Makarova ${ }^{1}$, Elvira Zvereva ${ }^{2}$, Yudenma Mongush $^{2}$ and Olga Zueva ${ }^{2, *}$ \\ ${ }^{1}$ Kazan Institute of Biochemistry and Biophysics, FRC Kazan Scientific Center of RAS, POB 30, \\ Kazan 420111, Russia \\ ${ }^{2}$ Kazan State Power Engineering University, 51, Krasnoselskaya str., Kazan, 420066, Russia
}

\begin{abstract}
The behaviour features of carbonate sludge which are a waste of water treatment process were studied in aqueous and oil environment. Based on the analysis of images of carbonate sludge particles in liquid media made by laser scanning confocal microscopes, the existence of nanostructures of various chemical compositions having several hundred nanometers in size, both single and forming porous conglomerate structures with a developed surface was revealed. The existence of such fragmentation of fine carbonate sludge powder particles in liquid media suggests that the mechanism of action of sludge additives is similar to that of nanoparticles. It was shown that the waste of the water treatment can be used as a cheap and environmentally friendly nanostructured material for industrial and environmental engineering, in particular, to improve the properties of hydrocarbon fuels. If necessary, the effect of carbonate sludge can be enhanced by changing its composition at the coagulation stage or by adding other nanoparticles. The data on the composition and texture characteristics of used sludge were given.
\end{abstract}

\section{Introduction}

One of the areas of modern industrial engineering is the development and optimization of technologies for the use of nanocomposite materials, i.e. materials which include nanoparticles. Nanoparticles are materials with sizes ranging from units to several hundred nanometers. The properties and behaviour of nanoparticles largely depend on their size, and besides their reactivity increases with decreasing size. The most active and at the same time highly toxic are artificially created particles (engineered nanoparticles, ENP) with size less than $50 \mathrm{~nm}[1,2]$.

It is known that the addition of nanoparticles leads to a radical transformation of material properties by modifying their morphology with virtually no change in their chemical composition. The physicochemical, operational, and environmental characteristics of nanocomposite materials can change, for example, due to appearance of additional structuring in the sample volume in presence of nanoparticles. Therefore, nanocomposite and nanostructured materials have many common features. In particular, the existence of opportunities for purposefully changing material structure by adding a certain type of

* Corresponding author: ostefzueva@ mail.ru 
nanoparticles in the required concentrations allows controlling their properties, which is very important for industrial and environmental engineering.

A similar approach is used in many applications of nanoparticles, including to improve the properties of diesel fuel. It turned out that the addition of small amounts of functional nanoscale structures to the fuel such as engineered nanoparticles of metals, oxides, carbides, nitrides [3-10], as well as carbon nanotubes [9-17], changes the properties of the fuel and can lead to an improvement in its operational and environmental characteristics. In our works [18-20], it was shown that the use of functional nanoscale structures can also be useful for improving the properties of heavy boiler fuel (fuel oil). Such additives alter the performance of the fuel and can lead to a decrease in the amount of harmful emissions into the atmosphere during the combustion of fuel oil [21].

In works [22,23] based on the analysis of literature data, we suggested that addition of nanoparticles leads to a change in the properties of not only fuel hydrocarbon systems, but also other liquid multicomponent systems. Such changes occur due to formation of microheterogeneous nanostructures in the bulk of multicomponent liquids. The purposeful variation of quantity and composition of small additives will allow changing the properties of complex systems in the required direction.

The advantages of using nanoparticles are huge. Despite the existence of many positive aspects of the impact of nanoparticles, their use must be approached with extreme caution, since it can be environmentally unsafe [24-28]. From the entire set of available nanostructured formations, we would like to choose the least aggressive forms, leading to changes in the microstructure and properties of composite materials in the right direction with minimal environmental consequences of their use. If necessary, to enhance the observed effect, the selected nanostructured additives can be mixed with a small amount of more active engineered nanoparticles. Such a technique will make it possible to take advantage of the synergistic properties of combined use of various nanoparticles.

We illustrate the above with the examples of additives to hydrocarbon fuels. Adding carbon nanotubes, which are widely used to create various nanocomposites, to the fuel improves the properties of boiler and diesel fuels. Moreover, in [15-17] it was shown that the best performance can be achieved when carbon nanotubes used together with engineered metal and metal oxides nanoparticles. However, it should be remembered that the use of metal and metal oxides nanoparticles can have a harmful effect on living organisms [24-28] and therefore requires strict environmental control.

In [19-21] we showed that instead of the mentioned nanoparticles carbonate sludge which is a waste of the coagulation and liming of natural waters at chemical water treatment, can be chosen as the main additive to heavy hydrocarbon fuel. The chemical composition of carbonate sludge is similar to the composition of nanoadditives used to improve the performance of diesel fuel, and to the composition of substances effective for binding sulfur and preventing corrosion of equipment in the fuel combustion zone. Industrial tests showed that the addition of carbonate sludge to fuel oil at a concentration of $0.1 \mathrm{wt}$. \% allowed to improve its viscosity properties and to achieve a significant reduction in emissions of sulfur oxides (by 36.5 wt. \%) in the atmosphere [21]. At the same time the composition of the ash has changed qualitatively. Most of the vanadium dioxide remained in the fuel combustion zone, accounting for $29.1 \mathrm{wt}$ \% ash residues [29]. An increase in vanadium dioxide in the ash clearly indicates about its decrease in the composition of the smoke emissions.

The use of carbonate sludge and materials based on it not only significantly improved the operational and environmental properties of fuels, but also gave good results in other areas of industrial engineering, for example, for wastewater treatment [30-33], biogas [34] and gas emissions [35-37], in the production of ceramic materials [38,39], polymer composites [40-42], porous materials [43], etc. The problems successfully solved with the 
use of carbonate sludge suggested that the mechanism of action of carbonate sludge particles is similar to that of nanoparticles [20].

This work is devoted to studying the composition, structure and properties of carbonate sludge as a cheap and environmentally friendly nanostructured substance for solving problems of industrial engineering. The use of carbonate sludge contributes to the solution of another environmental problem, namely the need for the disposal of waste generated during water treatment at thermal power plants in the processes of liming and coagulation. The accumulated volumes of sludge from water treatment, as well as their annual growth, are an unlimited resource in order to start mass and systematic use of carbonate sludge.

\section{Materials}

We used a dehydrated and ground in a fine grinding unit carbonate sludge of water treatment from Kazan TPP-1 with a final moisture content of about 1\%. For research, a finely dispersed fraction of carbonate sludge with a particle size of not more than 50 microns was taken. The total content of calcium compounds (in the form of calcite $\mathrm{CaCO} 3$ and portlandite $\mathrm{Ca}(\mathrm{OH}) 2$ ) and magnesium (in the form of brucite $\mathrm{Mg}(\mathrm{OH}) 2$ ) is not less than $80 \%, \mathrm{pH}=8.53$ [33]. The chemical composition and the ratio of the components of the sludge are determined by the chemical composition of raw water and the used coagulants. In the practice of clarification of source water, salts containing multiply charged cations, mainly salts of iron (or aluminum), are usually used as coagulants. The chemical composition of two samples of water treatment sludge from Kazan TTP-1 is presented in Table $1[37,44]$.

Table 1. The chemical composition of two samples of water treatment sludge [37,44].

\begin{tabular}{|c|c|c|c|c|c|}
\hline \multicolumn{6}{|c|}{ Mineral part - concentration of substances, wt. $\%$} \\
\hline \multicolumn{3}{|c|}{ Cations } & \multicolumn{3}{|c|}{ Anions } \\
\hline & Sample 1 & Sample 2 & & Sample 1 & Sample 2 \\
\hline $\mathrm{Ca}^{2+}$ & $76.56 \pm 11.3$ & $87 \pm 11.3$ & \multirow{2}{*}{$\mathrm{CO}_{3}^{2-}$} & \multirow{2}{*}{$71.7 \pm 10.6$} & \multirow{2}{*}{$81.5 \pm 10.6$} \\
\hline $\mathrm{Fe}^{3+}$ & $0.38 \pm 0.15$ & $0.44 \pm 0.15$ & & & \\
\hline $\mathrm{Mg}^{2+}$ & $9.7 \pm 2.2$ & $11 \pm 2.2$ & \multirow{2}{*}{$\mathrm{SO}_{4}^{2-}$} & \multirow{2}{*}{$5.7 \pm 0.85$} & \multirow{2}{*}{$6.5 \pm 0.85$} \\
\hline $\mathrm{Cu}^{2+}$ & $0.04 \pm 0.014$ & $0.05 \pm 0.014$ & & & \\
\hline $\mathrm{Ni}^{2+}$ & $0.008 \pm 0.003$ & $0.009 \pm 0.003$ & \multirow{2}{*}{$\mathrm{OH}^{-}$} & \multirow{2}{*}{$10.03 \pm 3.61$} & \multirow{2}{*}{$11.4 \pm 3.61$} \\
\hline $\mathrm{Zn}^{2+}$ & $0.033 \pm 0.013$ & $0.038 \pm 0.013$ & & & \\
\hline $\mathrm{Mn}^{2+}$ & $1.05 \pm 0.407$ & $1.2 \pm 0.407$ & \multirow{2}{*}{$\mathrm{SiO}_{3}{ }^{2-}$} & \multirow{2}{*}{$0.52 \pm 0.11$} & \multirow{2}{*}{$0.6 \pm 0.11$} \\
\hline $\mathrm{Cr}^{3+}$ & $0.001 \pm 0.0003$ & $0.001 \pm 0.0003$ & & & \\
\hline $\mathrm{Pb}^{2+}$ & $0.002 \pm 0.0003$ & $0.002 \pm 0.0003$ & \multirow{3}{*}{$\mathrm{PO}_{4}^{3-}$} & \multirow{3}{*}{ Is absent } & \multirow{3}{*}{ Is absent } \\
\hline $\mathrm{Cd}^{2+}$ & $0.22 \pm 0.08$ & $0.26 \pm 0.08$ & & & \\
\hline $\mathrm{Hg}^{2+}$ & Trace & Trace & & & \\
\hline
\end{tabular}

The content of organic carbon in dehydrated carbonate sludge is $11-12 \%$. The ash content of sludge annealed for 1 hour at a temperature of $400{ }^{\circ} \mathrm{C}$ in a muffle furnace is $89 \%$ [33].

Carbonate sludge does not contain highly toxic substances and has a low degree of harmful effects on the environment [41]. Wastewater treatment sludge belongs to the fifth hazard class (i.e. practically non-hazardous) [33]. Nevertheless, their storage at the sludge 
dumps causes some concern, as it leads to the alienation of large areas. In addition, there is a risk of violation of the chemical regime of soil water. The use of carbonate sludge as a secondary resource will make it possible to improve the environmental situation near thermal power plants.

\section{Methods}

Scanning laser confocal microscopes were used to obtain images of the microstructure of carbonate sludge particles and to assess their dispersibility in aqueous and oily media.

The LSM-510 Meta laser scanning confocal microscope (Carl Zeiss, Germany) based on the Axiovert 200M inverted microscope was used to obtain images in an aqueous medium. A small amount of sludge was placed in a drop of water on a glass slide and covered with a coverslip. For observation we used a HAL 100 halogen lamp of the LSM 510 META microscope, LD Plan-Neofluar 40x/0.6 objective. Microphotographs were taken with an AxioCam HRs camera (Zeiss, Germany).

Images of carbonate sludge distributed in a drop of immersion oil (type F, refractive index 1.52) were taken using a Leica TCS SP5 MP system (Leica Microsystems, Germany) with an objective (objective) HCX PL APO CS 63.0x1.40 OIL UV for research using laser confocal scanning microscopy and multiphoton microscopy.

For porosity studies nitrogen adsorption and desorption measurements at $77 \mathrm{~K}$ were carried out with ASAP 2020 MP instrument (Micromeritics). Before measurements sample was degassed by heating at $200{ }^{\circ} \mathrm{C}$ under vacuum $(8 \mu \mathrm{mHg})$ for 2 hours. Specific surface area of the sample was determined by applying the Brunauer-Emmett-Teller (BET) equation to the adsorption data obtained for the range of relative pressures of $0.05-0.30$ [45].Figures and tables, as originals of good quality and well contrasted, are to be in their final form, ready for reproduction, pasted in the appropriate place in the text. Try to ensure that the size of the text in your figures is approximately the same size as the main text (10 point). Try to ensure that lines are no thinner than 0.25 point.

\section{Experiment}

Images of carbonate sludge particles dispersed in an aqueous medium are presented in micrographs taken with a LSM-510 Meta laser scanning confocal microscope (Fig. 1)

Images of carbonate sludge particles distributed in an oily medium, made using a Leica TCS SP5 MP laser confocal microscope, are shown in Fig. 2 at various magnifications.

A study of the texture characteristics (specific surface area, pore size distribution, etc.) of carbonate sludge powder was carried out on the basis of the study of nitrogen adsorptiondesorption isotherm at $77 \mathrm{~K}$ (Fig. 3, left), obtained using an ASAP 2020 MP instrument (Micromeritics), and plotted as the BET graph in the framework of the Brunauer - Emmett - Teller model (Fig. 3, left) [45]. 

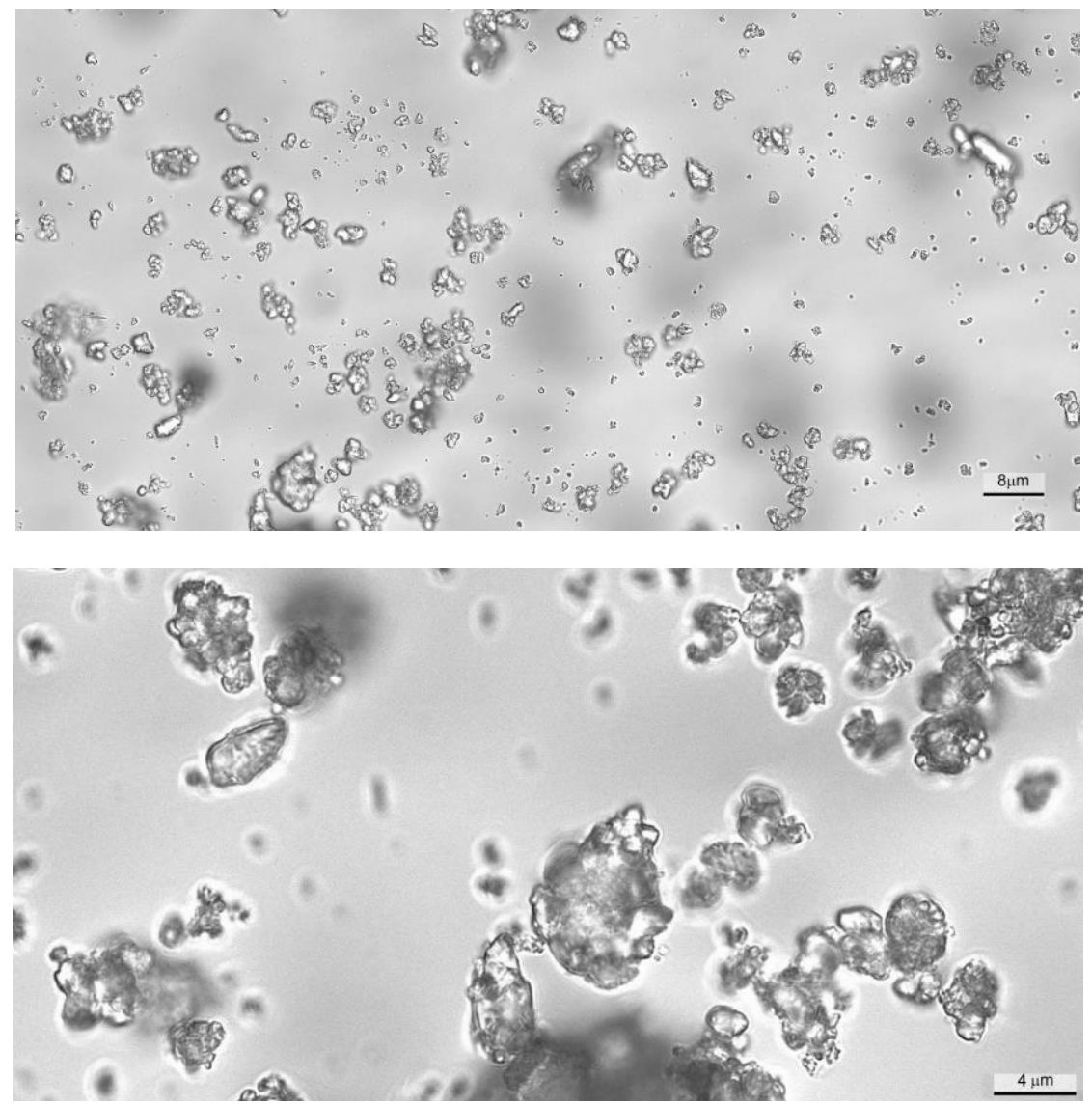

Fig. 1. Images of carbonate sludge dispersed in an aqueous medium at various magnifications.
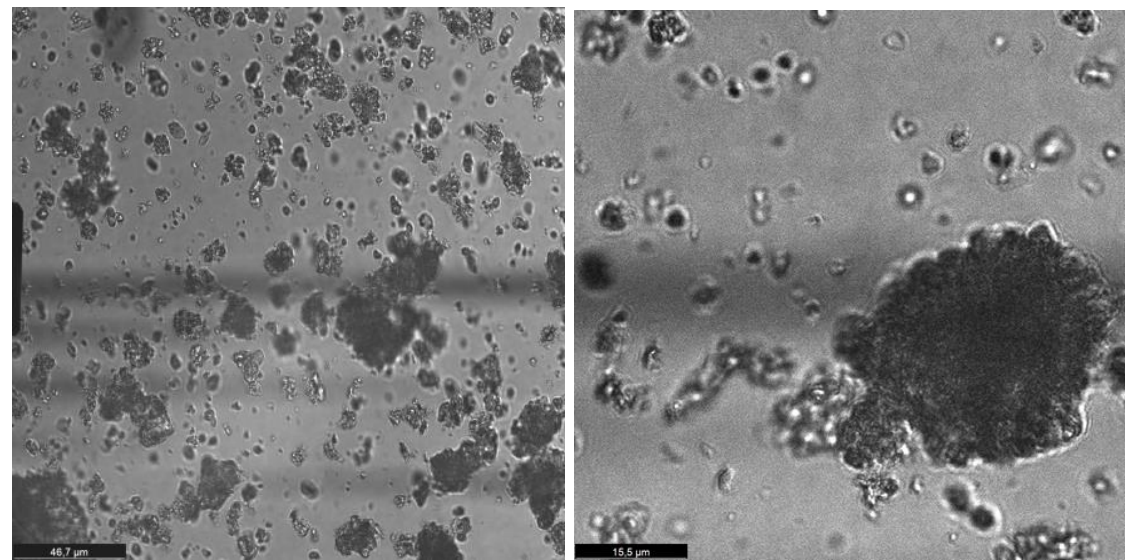

Fig. 2. Images of carbonate sludge dispersed in an oily medium at various magnifications. 

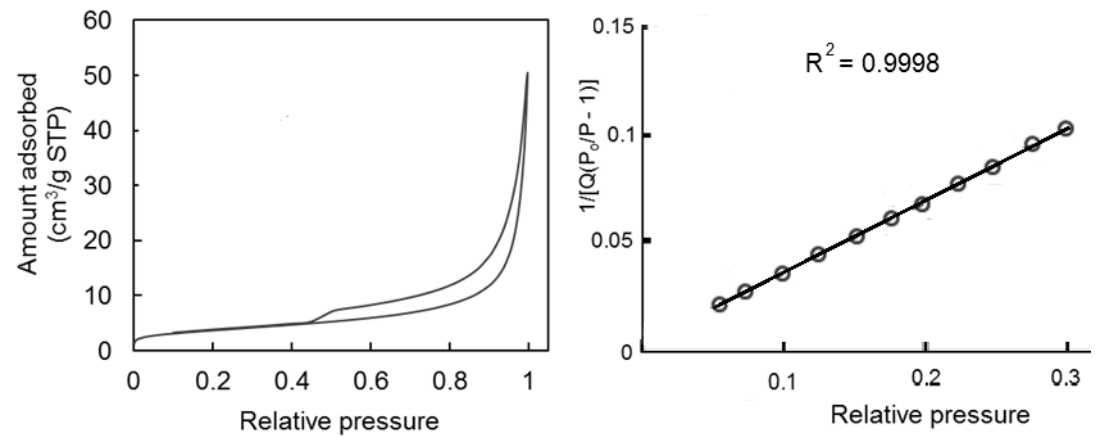

Fig. 3. Nitrogen adsorption-desorption isotherms at $77 \mathrm{~K}$ (left) and BET graph (right).

\section{Results and discussion}

Carbonate sludge as a product obtained by chemical deposition is characterized by a conglomerate structure of particles and a high specific surface. This waste has a complex of specific physicochemical properties determined by a diverse chemical composition (calcium carbonates, magnesium and iron hydroxides, etc.) and the extremely heterogeneous structure of its microparticles. Included in its composition compounds form a nano- and micro-dimensional structure, originally separated by water molecules. During the further coagulation process and during sludge dehydration, the structure of carbonate sludge particles becomes more complex. Small primary particles of finely dispersed carbonate sludge powder under the influence of surface forces stick together in more or less dense secondary aggregates with a developed surface and porous structure. This fact is indicated by images of carbonate sludge particles distributed in a drop of water (Fig. 1) and oil (Fig. 2).

In addition, it can be seen that in water (Fig. 1), the initial particles, whose size in dehydrated sludge reached $50 \mu \mathrm{m}$, decomposed into many much smaller formations, the minimum size of which with a laser confocal microscope with a resolution limit of $\sim 250$ $\mathrm{nm}$ cannot be defined. Nevertheless, it can be noted that even formations smaller than $1 \mu \mathrm{m}$ have a complex structure corresponding to aggregates of smaller adhering particles. An increase in dispersibility of microparticles in aqueous suspensions indicates the hydrophilic nature of the surface and the presence of partial solubility in water of both mineral and, possibly, organic components.

In an oily environment, the average size of individual particles increases. Apparently, this is due to the low hydrophobicity of the surface of the microparticles and to the lower solubility of individual components of an inorganic nature in oil. Nevertheless, nanoscale structures also exist in an oil medium. Larger micro-sized particles show a conglomerate structure, which is corresponded the presence of pores, and a developed surface. However, it should be noted that when carbonate sludge is added to heavy fractions of fuel oil, which always contain water impurities, hydrophilic particles of sludge will primarily interact with water pushed by hydrophobic hydrocarbon compounds into the near-surface layers of the fuel and with water adsorbed from the air. Therefore, even when mixed with fuel, the dispersibility of carbonate sludge will more closely correspond to that observed in an aqueous rather than an oil environment.

Fragmentation of the initial particles of carbonate sludge into smaller particles in aqueous and oily media suggests that the mechanism of action of particles of carbonate sludge is similar to the action of nanoparticles used to improve the performance of diesel fuel [3-10]. 
Specific surface area, sample porosity, pore volume, pore size distribution allows determining the method of gas adsorption and capillary condensation. This method successfully complements the microscopy method, giving more complete information about the sample. The most commonly used adsorbate is nitrogen. The specific surface area can be calculated from nitrogen adsorption and desorption measurements using a number of classical models. Commonly used model is Bruner - Emmett - Teller model.

In carbonate sludge powder, the gaps between the primary particles inside the secondary aggregates and the gaps between the secondary aggregates form a porous system in which the shape and size of individual pores depend on the shape and size of the primary and secondary particles. From the type of adsorption-desorption isotherms, it can be concluded about the surface area, the porosity of the adsorbing body, and the nature of the interaction between the adsorbent and adsorbate.

The shape of the adsorption-desorption isotherm experimentally obtained on the ASAP 2020 MP instrument (Micromeritics) for the carbonate sludge powder is shown in Fig. 3 (left). According to the IUPAC classification for adsorption - desorption isotherms the obtained isotherm having a hysteresis loop reflects the course of capillary condensation in mesopores (in pores with diameters from 2 to $50 \mathrm{~nm}$ ). However, the fact that the adsorption does not reach saturation and the type of the hysteresis loop indicate that the porous system is highly disordered, and in general, the data obtained cannot be used to correctly evaluate both the distribution of pore size and the total pore volume.

Using the BET method allows us to calculate the value of the monolayer capacity in the case of polymolecular adsorption. To calculate the specific surface, a linear form of the BET equation is used and a BET graph is constructed (Fig. 3, right), in which the value $1 /\left[Q\left(P_{0} / P-1\right)\right]$, determined by the mass of adsorbate $Q$ per $1 \mathrm{~g}$ of adsorbent, linearly depends on relative pressure $P / P_{0}$ [45]. Here $P$ and ${ }_{0}$ are the equilibrium vapor pressure and saturated vapor pressure of the adsorbate at the adsorption temperature. The calculation of the specific surface area of the carbonate sludge powder by BET gives a value of $13.07 \pm$ $0.06 \mathrm{~m}^{2} / \mathrm{g}$.

Data obtained from nitrogen adsorption and desorption measurements indicate that carbonate sludge in the form of a dry powder is not a highly structured substance. That is why its use, for example, as an adsorbent, requires additional modification of the properties of its surface [30-33].

It should be noted, however, that the calculated specific surface area of $13.07 \pm 0.06$ $\mathrm{m}^{2} / \mathrm{g}$ corresponds to dehydrated carbonate sludge powder. When using carbonate sludge in liquid media, it should be classified as nanostructured, since due to fragmentation of the sludge particles and increase in the dispersibility of the sample, the actual value of the specific surface area will be much larger and the particle sizes will be much smaller.

An important fact is that the carbonate sludge preparation technique allows one to control its composition, and hence its properties, by adding the necessary compounds, for example, salts of certain metals, to solutions. In the process of chemical precipitation, embedded salts, just like the initial ones, will first settle in the form of nanoparticles, and at the next stage they will create conglomerate structures of a more complex composition. In liquid media, conglomerate structures of the modified carbonate sludge powder will partially decompound into smaller nanostructured formations that change the properties of heterogeneous systems upon their introduction. In particular, one of the ways to change the operational properties of the fuel in the right direction can be modification (enrichment) of carbonate sludge. 


\section{Conclusion}

In this paper the behavior features of carbonate sludge microparticles which is a waste of the water treatment process, in aqueous and oily media have been studied. Analysis of images of carbonate sludge particles in liquid media, performed by scanning confocal microscopy (LSM-510 Meta laser, Carl Zeiss, Germany and Leica TCS SP5 MP, Leica Microsystems, Germany), revealed the existence of nanostructures of various chemical compositions several hundred nanometers in size (or maybe less), both free and adhered to each other with the formation of porous structures. This means that the initial conglomerate microparticles of carbonate sludge in aqueous and oily media decomposed into smaller particles having a size of several hundred nanometers. Such fragmentation of particles of finely dispersed carbonate sludge powder in liquid media suggests that the mechanism of action of sludge additives is similar to that of nanoparticles. At the same time, carbonate sludge particles cannot be assigned to engineered nanoparticles, their structure, size and properties are more consistent with natural formations. This means that the waste of the water treatment process can be used as a cheap and environmentally friendly nanostructured substance for industrial and environmental engineering.

Thus, carbonate sludge is one of the least aggressive forms of nanostructured formations, the addition of which can lead to changes in the microstructure and properties of composite materials in the right direction with minimal environmental consequences of their use. If necessary, to enhance the effect required for solving each specific problem, this additive can be mixed with a small amount of nanoadditives of more active forms, such as, for example, carbon nanotubes. Such a technique will make it possible to take advantage of the synergistic properties of the combined use of various nanoparticles.

In our opinion, one of the new areas of carbonate sludge application may be the creation of nanocomposite hydrogels based on natural biopolymers with enhanced strength characteristics. Such hydrogels can be used to solve various tasks of water purification, controlled application of pharmacological agents, nutrients and plant protection products, improvement of soil structure, etc.

This work was partly financially supported by the RFBR (projects numbers 19-38-90085). A. Makarova thanks the State Assignment to the Kazan Scientific Center of the Russian Academy of Sciences (preparing of samples for confocal microscopy) and the grant of RF President for young scientists (confocal data analysis). The authors thank Dr. M. Ageeva and Dr. D. Samigullin from KIBB FRC Kazan Scientific Center of RAS for experiments with confocal microscopy.

\section{References}

1. S.J. Klaine, P.J.J. Alvarez, et al., Environmental Toxicology and Chemistry, 27, 1825 (2008) DOI: 10.1897/08-090.1

2. B. Nowack, T.D. Bucheli, Environ. Pollut., 150, 5 (2007) DOI: 10.1016/j.envpol.2007.06.006

3. J.S. Basha, R.B. Anand, JRSE, 3, 023106 (2011) DOI: 10.1063/1.3575169

4. T. Shaafi, K. Sairam, A. Gopinath, G. Kumaresan, R. Velra, Renew. Sust. Energ. Rev., 49, 563 (2015) DOI: 10.1016/j.rser.2015.04.086

5. J.S. Basha, R.B. Anand, J. Braz. Soc. Mech. Sci., 35, 257 (2013) DOI: 10.1007/s40430-013-0023-0

6. M.A. Lenin, M.R. Swaminathan,G. Kumaresan, Fuel, 109, 362 (2013) DOI: 10.1016/j.fuel.2013.03.042 
7. G.R. Kannan, R. Karvembu, R. Anand, Applied Energy, 88, 3694 (2011) DOI: 10.1016/j.apenergy.2011.04.043

8. W.M. Yang, H. An, S.K. Chou, S. Vedharaji, R. Vallinagam, Fuel, 104, 726 (2013) DOI: 10.1016/j.fuel.2012.04.051

9. H.S. Jung, A. Miller, K. Park, D.B. Kittelson, J. Air. Waste Manag. Assoc., 63, 1199 (2013) DOI: 10.1080/10962247.2013.812048

10. K Heydari-Maleney, A Taghizadeh-Alisaraei, B. Ghobadian, A. AbbaszadehMayvana, Fuel, 196, 110 (2017) DOI: 10.1016/j.fuel.2017.01.091

11. A.L. El-Seesy, A.K. Abdel-Rahman, M. Bady, S. Ookawara, Energy Procedia, 100, 166 (2016) DOI: 0.1016/j.egypro.2016.10.160

12. S.H. Hosseini, A. Taghizadeh-Alisaraei, B. Ghobadian, A. Abbaszadeh-Mayvan, Renewable Energy, 111, 201 (2017) DOI: 10.1016/j.renene.2017.04.013

13. N. Singh, R.S. Bharj, Proc. I. Mech. E. Part C: Journal of Mechanical Engineering Science, 2302046 (2015) DOI: 10.1177/0954406215586021

14. A.L. El-Seesy, A.K. Abdel-Rahman, M. Bady, S. Ookawara, Energy Conversion and Management, 135, 373 (2017) DOI: 10.1016/j.enconman.2016.12.090

15. G. Najafi, Fuel, 212, 668 (2018) DOI: 10.1016/j.fuel.2017.10.001

16. M. Ghanbari, G. Najafi, B. Ghobadian, T. Yusaf, A.P.Carlucci, M.Kiani Deh Kiani, Fuel, 202, 699 (2017) DOI: 10.1016/j.fuel.2017.04.117

17. M. Mirzajanzadeh, M. Tabatabaei, M. Ardjmand, A. Rashidi, B. Ghobadian, M. Barkhi, M. Pazouki, Fuel, 139, 374 (2015) DOI: 10.1016/j.fuel.2014.09.008

18. E. R. Zvereva, O. S. Zueva, R. V. Khabibullina, G. R. Mingaleeva, G. R. Akhmetvalieva, D. R. Salikhzyanova, Z. F. Khatmullina, Chemistry and Technology of Fuels and Oils, 52, 488 (2016) DOI: 10.1007/s10553-016-0734-X

19. E.R. Zvereva, O.S. Zueva, R.V. Khabibullina, A.O. Makarova, Journal of Engineering and Applied Sciences, 11, 2950 (2016) DOI: 10.3923/jeasci.2016.2950.2954

20. E. R. Zvereva, R. V. Khabibullina, A. O. Makarova, G. R. Akhmetvalieva, F.I. Burganova, D. V. Ermolaev, O. S. Zueva, Petroleum Chemistry, 59, 106 (2019) DOI: 10.1134/S0965544119010158

21. E.R. Zvereva, A.V. Dmitriev, M.F. Shageev, G.R. Akhmetvalieva, Therm. Eng., 64, 591 (2017) DOI: 10.1134/S0040601517080110

22. E.R. Zvereva, A.O. Makarova, O.S. Zueva, IOP Conference Series: Earth and Environmental Science, 272, No 022238 (2019) DOI: 10.1088/1755$1315 / 272 / 2 / 022238$

23. E.R. Zvereva, A.O. Makarova, O.S. Zueva, Solid State Phenomena, 284, 767 (2018) DOI: 10.4028/www.scientific.net/SSP.284.767

24. M. Kumari, S.P. Singh, S. Chinde, M.F. Rahman, M. Mahboob, P. Grover, Int. J. Toxicol., 33, 86 (2014) DOI: 10.1177/1091581814522305

25. N.B. Turan, H.S. Erkan, O.G. Engin, M.S. Bilgili, Process Safety and Environmental Protection, 130238 (2019) DOI: 10.1016/j.psep.2019.08.014

26. R. Hardman, Environ. Health Perspect., 114, 165 (2005) DOI: 10.1289/ehp.8284

27. M.A. Maurer-Jones, I.L. Gunsolus, C.J. Murphy, C.L. Haynes, Anal. Chem., 85, 3036 (2013) DOI: 10.1021/ac303636s

28. J.R. Peralta-Videa, L. Zhao, M.L. Lopez-Moreno, G. de la Rosa, J. Hong, J.L. GardeaTorresdey, J. Hazard. Mater., 186, 1 (2011) DOI: 10.1016/j.jhazmat.2010.11.020 
29. E.R. Zvereva, O.S. Zueva, K.K. Gilfanov, V.P. Tutubalina, IOP Conference Series: Earth and Environmental Science, 337, No 012060 (2019) DOI: 10.1088/1755$1315 / 337 / 1 / 012060$

30. L.A. Nikolaeva, R.Ya. Iskhakova, Therm. Eng., 64, 458 (2017) DOI: 10.1134/S0040601517060040

31. L.A. Nikolaeva, M.A. Golubchikov, A.R. Minneyarova, Chem. Petrol. Eng., 53, 806 (2018) DOI: 10.1007/s10556-018-0426-y

32. L.A. Nikolaeva, A.G. Laptev, R.Ya. Iskhakova, Water Resour., 45, 231 (2018) DOI: 10.1134/S0097807818020136

33. L.A. Nikolaeva, R.Y. Iskhakova, Therm. Eng., 64, 458 (2017) DOI: 10.1134/S0040601517060040

34. O.W. Awe, Y. Zhao, A. Nzihou, et al., Waste Biomass Valor, 8, 267 (2017) DOI: 10.1007/s12649-016-9826-4

35. H.P. Xuan, D.P. Minh, M.G. Martínez, A. Nzihou, P. Sharrock, Ind. Eng. Chem. Res. 54, 4915 (2015) DOI: 10.1021/acs.iecr.5b00764

36. L.A. Nikolaeva, Therm. Eng., 60, 244 (2013) DOI: 10.1134/S0040601513040095

37. L.A. Nikolaeva., A.N. Khusnutdinov, Therm. Eng., 65, 575 (2018) DOI: 10.1134/S0040601518080049

38. C.M.F. Vieira, S.N. Monteiro, Matéria (Rio de Janeiro), 14, 881 (2008) DOI: 10.1590/S1517-70762009000300002

39. B.E.E. Hegazy, H.A. Fouad, A.M. Hassanain, Australian Journal of Basic and Applied Sciences, 6, 453 (2012) ISSN 1991-8178

40. S. Kashyap, D. Datta, Materials Today: Proceedings, 4A, 2946 (2017) DOI: 10.1016/j.matpr.2017.02.176

41. Nanomaterials and Nanotechnologies in Power Industry, Ed. by E. V. Shamsutdinov and O. S. Zueva (KGEU, Kazan, 2014), ISBN 978-5-89873-421-3 [in Russian].

42. K. Ahmed, S.S. Nizami, N.Z. Riza, J Adv Res. 5, 165 (2014) DOI: 10.1016/j.jare.2013.01.008

43. Y. Bian, Q. Yuan, G. Zhu, B. Ren, A. Hursthouse, P. Zhang, Int. J. Polym. Sci., 2018, No 8320609 (2018) DOI: 10.1155/2018/8320609

44. L.A. Nikolaeva, A.R. Minneyarova, Therm. Eng, 66, 372 (2019) DOI: 10.1134/S0040601519050069

45. M. Thommes, K. Kaneko, A.V. Neimark, J.P. Olivier, F. Rodriguez-Reinoso, J. Rouquerol, K.S. Sing, Pure Appl. Chem., 87, 1051 (2015) DOI: 10.1515/pac-20141117 